\title{
Drei neue Handschriften des sog. Chronicon hungarico-polonicum
}

Das sog. Chronicon hungarico-polonicum, das in der jüngeren Vergangenheit verstärkt die Aufmerksamkeit von Historikerinnen und Historikern gefunden hat ${ }^{1}$, ist ein wohl zwischen 1220 und 1235 am Hof des 1215/16 zum König von Halič gekrönten ungarischen Königssohn Koloman, entstandener Text, der nach 1241 mit seiner Witwe Salomea nach Polen gelangte. Die Chronik erzählt die Geschichte der Ungarn und ihres Königs Aquila (d.i. Attila) von ihrem Auszug aus der östlichen Ungaria bis zur Herrschaft des Königs Ladislaus. Die Chronik liegt in zwei Redaktionen vor, deren erste und längere eher einen historischen Charakter hat, während die zweite und kürzere hagiographisch Züge trägt; sie wird auch als Vita s. Stephani epitomata (Biblioteca Hagiographica Latina [weiter: BHL] 7922) geführt. Das Chronicon gliedert sich in drei Teile. Im ersten, nur in der längeren Redaktion erhaltenen Teil, wird die Wanderung der Ungarn durch Europa nach Kroatien und in die Sclavonia beschrieben, in der Attila einen Ungarn genannten Staat gegründet habe (in einer sehr reduzierten Form ist dies auch zu Beginn der Vita s. Stephani epitomata überliefert). Dieser Teil basiert teils auf den verlorenen Gesta Ungarorum, teils auf kroatischen und ungarischen Quellen. Der zweite Teil, der in beiden Versionen in unterschiedlicher Länge erzählt wird, berichtet basierend auf der Vita s. Stephani des Hartvik (BHL 7921) die Geschichte der Konversion König / Herzog Yesses (Gézas) durch seine (apokryphe) polnische Ehefrau Adelheid und das Leben ihres Sohnes Stephan. Der dritte und letzte Teil weicht in beiden Versionen stark voneinander ab. Nur in der längeren Rezension wird die fabelhafte Geschichte von Stephans Söhnen erzählt, die nach Polen geflohen sein sollen, und sodann folgt die Geschichte der polnischen Intervention in Ungarn mit der anschließenden glücklichen Regie-

\footnotetext{
${ }^{1}$ R. Grzesik, Chronicon hungarico polonicum, in: Encyclopedia of Medieval Chronicle, Vol. 1, 348 f.; A. Quéret-Podesta, The influence of the so-called Hungarian-Polish chronicle on the Silesian medieval chronicles, „Slezský sborník”, 108, 2010, S. 273-278; Ders., Vom Ungarn der Arpáden zum Polen der Piasten. Zur Entstehung und zum Schicksal der sogenannten Ungarisch-polnischen Chronik, in: Mittelalterliche Eliten und Kulturtransfer östlich der Elbe: Interdisziplinäre Beiträge zu Archäologie und Geschichte im mittelalterlichen Ostmitteleuropa, hrsg. von A. Klammt, Göttingen 2009, S. 69-80; Żywot św. Stefana króla Wegier czyli Kronika wegiersko- polska, wyd. Ryszard Grzesik, Warszawa 2003; Ders., Kronika węgiersko-polska. Z dziejów polsko-węgierskich kontaktów kulturalnych w średniowieczu, Poznań 1999; M. Homza, Mulieres suadentes. Presviedčajúce ženy, Bratislava 2002; Ders., The System of Proper Personal Names in the Hungarian-Polish Chronicle, in: East Central Europe at the turn of the 1st and 2nd millennia, ed. V. Múcska, Acta historica posoniensia 2, Bratislava 2002, S. 49-69; J. Csákó, A magyar-lengyel krónika és a hazai elbeszélő hagyomány, „Századok”, 148, 2014, S. 287-334; R. Grzesik, Legitimierungsfunktion der ungarisch-polnischen Chronik, in: The Medieval Chronicle. Proceedings of the 1st International Conference on the Medieval Chronicle, Driebergen/Utrecht, 13-16 July 1996, ed. E. Kooper, Amsterdam 1999, S. 144-154.

Für einige wertvolle Hinweise möchte ich mich an dieser Stelle ganz herzlich bei dem wohl besten Kenner der Chronik, Herrn Prof. Dr. Ryszard Grzesik sowie bei Herrn Prof. Ernst-Dieter Hehl bedanken.
} 
rung des hl. Ladislaus. Die kürzere Rezension hat statt dessen eine wiederum auf Hartwik basierende Fortsetzung der Vita s. Stephani mit einigen Wundern.

Während die Chronik im mittelalterlichen Ungarn nicht rezipiert wurde, schöpfen zahlreiche insbesondere kleinpolnische und schlesische Quellen aus ihr, wie etwa die Vita maior sancti Stanislai des Vinzenz von Kielcza. In den schlesischen und kleinpolnischen Annalen des ausgehenden 13. bzw. des 14.-15. Jahrhunderts ist die Geschichte der Hochzeit Adelheids und, wie auch schon bei Vinzenz, eine Erzählung von der ungarisch-polnischen Rivalität um die vom Papst verliehene Krone enthalten. Dabei lag den polnischen Annalisten wohl meistens kein originales Manuskript der Chronik vor, sondern sie benutzten vermutlich eine Überarbeitung ${ }^{2}$.

Die editio princeps der compositio maior besorgte 1823 Hipolit Kownacki, weitere Ausgaben erfolgten durch Stephanus Ladislaus Endlicher, Stanisław Piłat, Marianus [Matthias] Florianus und Jozsef Deér ${ }^{3}$, die editio princeps der compositio minor besorgte 1897 Wojciech Kętrzyński ${ }^{4}$. Die heute gültige Edition ist die dreispaltig angelegte Ausgabe von Béla Karácsonyi, die alle bisher bekannten Handschriften berücksichtigt, wobei die mittlere Spalte den gemeinsamen Textbestand enthält, die jeweils Linke das Sondergut der compositio maior und die Rechte das der compositio minor. Seine Siglen werden im Folgenden benutzt ${ }^{5}$. Eine weitere Edition, die die Chronik als Mischtext präsentiert, wurde samt einer slowakischen Übersetzung zuletzt von M. Homza vorgelegt ${ }^{6}$.

\section{Bisher bekannte Handschriften}

Vom Chronicon Hungarico-Polonicum waren bisher folgende Handschriften bekannt.

$\mathrm{Z}=$ Warschau, BN, BOZ 28. Die beste Hs. der längeren Redaktion ist die des Codex Zamoyski, eine Pergamenthandschrift aus dem Ende des 14. Jh.s mit den Maßen 25,4 cm $\times 18 \mathrm{~cm}$ auf 97 Folioseiten, geschrieben in einer ausgeprägten gotischen Minuskel, die auf eine Verbindung zu einer italienischen Schule hinzuweisen scheint. Die Chronik beginnt auf fol. $90^{\mathrm{r}}$ und endet auf fol. 96 ${ }^{\mathrm{v}}$. Die Handschrift enthält außerdem eine Lebensbeschreibung Alexanders des Großen, die Chronik des Gallus anonymus, die sog. Spominki krakowskie (Krakauer Notizen) zu den Jahren 1439 und 1437-1447, eine Überarbeitung des 14. Jh.s der Vita et miracula sancti Stanislai des Vinzent von Kielcza (inc. Tradunt), das Rocznik Traski (Annalen des Traska) und einige andere Texte.

C $($ S bei Grzesik $)=$ Krakau, Muzeum Narodowe, Zbiory Czartoryskich, Codex Cracovienis Czartoryskianus 1310. Eine in Polen angefertigte Kopie der Chronik aus der zweiten Hälfte des 15. Jahrhunderts liegt im Codex Cracovienis Czartoryskianus 1310 vor, der auf 949 Seiten verschiedene Werke enthält, die vor allem aus $\mathrm{Z}$ abgeschrieben wurden; die ungarisch-polnische Chronik befindet sich auf den fol. $349^{r}-359^{\text {r7 }}$.

\footnotetext{
2 A. Quéret-Podesta, Vom Ungarn der Arpáden zum Polen der Piasten. Zur Entstehung und zum Schicksal der sogenannten Ungarisch-polnischen Chronik, in: Mittelalterliche Eliten, S. 69-80, hier bes. S. 77.

${ }^{3}$ H. Kownacki, Kronika Wegierska na poczatku wieku XII; Kronika czeska na początku wieku XI, Warszawa 1823; S.L. Endlicher, Chronica hungarorum, in: Rerum hungaricarum Monumenta Arpadiana, Sangalli 1849, S. 60-82; S. Piłat, Kronika weggiersko-polska, in: MPH 1, S. 495-515. — Matthias Florianus, Vita Sanctorum Stephani regis et Emerici ducis, Historiae Hungaricae Fontes Domestici, Pars Prima: Scriptores I, Lipsiae 1881, S. 70-79; J. Deér, Scriptores Rerum Hungaricarum vol. 2, Budapestini 1938, S. 299-320.

${ }^{4}$ W. Kętrzyński, O kronice węgiersko-polskiej (Vita sancti Stephani, regis Ungariae, Ungarico-Polona), RAU whf, 34, 1897, S. 365-373.

5 B. Karácsonyi, Chronica hungaro-Polonica, pars I (Textus cum varietate lectionum), Acta Historica Universitatis Szegedensis de Attila Józef nominatae, t. 26, Szeged 1969.

${ }^{6}$ M. Homza, Uhorsko-pol'ská kronika. Nedocenený prameň k dejinám strednej Európy, Bratislava 2009.

${ }^{7}$ Eine genauere Beschreibung gibt: W. Drelicharz, Annalistyka małopolska XIII-XV wieku. Kierunki rozwoju wielkich roczników kompilowanych, Kraków 2003, S. 44-56.
} 
$\mathrm{K}=$ Verloren ist heute der Codex Varsovianus Krasinskianus [der Krasiński-Bibliothek in Warschau] 83, eine Papierhandschrift des 16. Jahrhunderts, die im Zweiten Weltkrieg verschollen ist. Nach der einzigen Beschreibung soll die Chronik auf den fol. $7^{\mathrm{r}-\mathrm{v}} \mathrm{zu}$ lesen gewesen sein.

$\mathrm{W}=$ Breslau, Ossolineum, Codex Vratislaviensis Ossolianus I.818 Eine weitere Papierhandschrift des 15. Jahrhunderts ist der Codex Vratislaviensis Ossolinianus I.818, der vor allem theologische Texte enthält. Auf fol. 177v liegen die ersten Zeilen der Chronik wie in der längeren Redaktion $(Z)$ vor.

$\mathrm{O}=$ Breslau, Ossolineum, Codex Vratislaviensis Ossolianus II. 1944.

Dabei handelt es sich um eine 433 fol. starke Papierhandschrift, die mit Passionale überschrieben ist. Die Handschrift datiert in das 15. Jahrhundert, wie der Herausgeber meinte, probabiliter ex Polonia oriundus. Die Handschrift ist eine in polnischer Umgebung entstandene Variante der Legenda aurea und umfasst 211 Legenden. Sie enthält auf den fol. $371^{\mathrm{r}}-375^{\mathrm{r}}$ den bisher einzigen bekannten Text der Vita s. Stephani regis Ungarie (BHL 7922), also der kürzeren Redaktion der ungarisch-polnischen Chronik, und ist daher die Grundlage für den Vergleich mit den hier vorgelegten beiden neuen Handschriften. Daneben enthält sie als Anhang eine Reihe weiterer Heiliger teils aus Kleinpolen (Stanislaus), teils aus Schlesien (Hedwig), teils aus Böhmen (Adalbert, Ludmilla, Wenzel, Prokop), teils aus Ungarn (Elisabeth, Emmerich) ${ }^{8}$.

\section{Neue Handschriften}

Außer in den vorgenannten bekannten Handschriften liegt die kürzere Fassung des Chronicon, bzw. die Vita s. Stephani (BHL 7922) noch in zwei bisher übersehenen Codices vor.

Es handelt sich dabei um eine Prager (im Folgenden P) und um eine Magdeburger Handschrift (im Folgenden M).

Die Prager Handschrift ${ }^{9}$, die heute unter der Signatur XIV.A.7 in der Nationalbibliothek in Prag (Národní knihovna České republiky) aufbewahrt wird, entstand vermutlich in Böhmen selbst im letzten Drittel des 14. Jh.s, wohl um 1380, aber nicht vor 1366 und nicht nach 1400. Es handelt sich dabei um eine sorgfältig zweispaltig geschriebene Pergamenthandschrift mit I+376 Folioseiten in einem Format von $38 \mathrm{~cm} \times 27 \mathrm{~cm}$ und einem Textspiegel von $25 \mathrm{~cm} \times 17,5 \mathrm{~cm}$ mit etwa 40 Zeilen pro Seite.

Die Handschrift ist eine auf der Legenda aurea basierende Sammlung von Viten, die größtenteils dem Verlauf des Kirchenjahres entsprechend eingetragen sind. Gegen Ende der Hs. finden sich nur die Legenden der bekanntesten Heiligen, während zu Beginn auch die Leben weniger bekannter Heiliger eingetragen sind. Die Vorlage der Hs. entstand wohl als Kompilation verschiedener Quellen. Der kompilatorische Charakter wird dadurch besonders deutlich, dass einige Texte doppelt vorhanden sind. Bei der Erstellung des Textbestandes wurde nicht auf eine Einheitlichkeit des Umfangs geachtet, vielmehr sind einige Texte eher kurze martyrologische Notizen, andere sind von großem Umfang, die längsten Texte betreffen die hll. Gallus und Livinus. Insbesondere enthält die Handschrift eine Reihe böhmischer Heiliger. So finden sich hier die Ludmillalegende Fuit in provincia, die Translacio Ludmile, die Vita und Translatio des hl. Wenzels, die Vita des hl. Prokop und die Vita der hll. fünf Brüder (De quinque fratribus) (BHL 1148), in der Version des Cosmas von Prag.

Der Umstand, dass die Legenda quemadmodum bzw. Tempore Michaelis imperatoris fehlt, lässt die Annahme zu, die Vorlage sei vor der Mitte des 14. Jahrhunderts entstanden ${ }^{10}$.

\footnotetext{
${ }^{8}$ R. Grzesik, Chronicon hungarico polonicum, 348f.; Ders., Kronika wegiersko-polska.

${ }^{9} \mathrm{http} / / /$ www.manuscriptorium.com/apps/main/mns_direct.php?docId=rec1283761530_44 [Zugang: 22.06.2015], vgl. Josef Truhlár, Catalogus codicum manu scriptorum latinorum, qui in c. r. bibliotheca publica atque Universitatis Pragensis asservantur, t. 2, Pragae 1906, č. 2419, S. 276-277; E. Urbánková, Rukopisy a vzácné tisky pražské Universitni knihovny, Praha 1957, č. 55, S. 19; J. Krása, Rukopisy Václava IV, Praha 1971, S. 252.

10 Vgl. Tempore Michaelis imperatoris, S. 255-257; Legenda Quemadmodum, S. 289-291, in: Magnae Moraviae Fontes historici II. Textus biographici, hagiographici, liturgici, Brno 1967. Zu der Verbreitung dieser Teile in den Legenda aurea-
} 
Außer der auf $138^{\text {va }}-142^{\text {ra }}$ aufgezeichneten Vita sancti Stephani regis Ungarie weist auch die Vita Henrici regis Ungarie (BHL 2529b) auf ungarische Beziehungen der Vorlage hin; schlesische Beziehungen sind durch einen Sermo venerabilis Clementis pape de canonizacione Hedwigis und die Minor legenda de sancta Hedwigis (BHL 3767d) $\left(253^{\mathrm{r}}-257^{\mathrm{r}}\right)$ belegt $^{11}$.

Die andere Handschrift (Ms. Magdeb. 138) umfasst den Sommerteil des großen Magdeburger Legendars ${ }^{12}$. Es handelt sich dabei um eine zweispaltige Papierhandschrift aus dem Bestand des Domgymnasiums mit IX, 443 Blättern im Format $31,5 \mathrm{~cm} \times 21,5 \mathrm{~cm}$, wobei der Schriftraum etwa $25 \mathrm{~cm} \times 15 \mathrm{~cm}$ beträgt. Geschrieben wurde sie von mehreren Händen, darunter auch dieselben wie in Ms. Magdeb. 26 (mit dem Winterteil des Legendars), das mit 1459 datiert ist und auch auf dem gleichen Papier geschrieben wurde, weshalb auch Ms. Magdeb. 138 im gleichen Zeithorizont entstanden sein dürfte. Beide Handschriften sind entweder in Magdeburg selbst, oder aber in dessen näherem Umfeld entstanden, wofür das Vorhandensein lokaler Legenden und Anekdoten spricht. Das Legendar war vermutlich für den Gebrauch des Magdeburger Domstifts bestimmt. Außer der Vita des hl. Stephan enthält die Handschrift die Materia Langobardorum et cronica imperatorum, eine Bearbeitung der lateinischen Fassung der Sächsischen Weltchronik, in der sich auch ein Bericht über ein großes Ritterspiel findet, das 1278 in Magdeburg stattgefunden haben soll, ferner befindet sich darin ein Brief eines gewissen Simon aus Konstantinopel mit einem Bericht über den Fall der Stadt 1453. Das Legendar ist eine Zusammenstellung verschiedener Martyrologien und Legendare, Grundlage ist die Legenda aurea, außerdem die Martyrologien des Usuardus und des Ado Viennensis, das Speculum historiale des Vinzenz von Beauvais, der Liber pontificalis und "vermutlich auch ein Legendarium Halberstatense « ferner unbekannte Quellen. Mehrere Texte könnten auf eine Verbindung mit Böhmen bzw. Prag hinweisen, ein Umstand der U. Winter nicht weiter aufgefallen ist. Zunächst ist da eine »in Anlehnung an die Vita s. Adalberti des Brun von Querfurt verfasste Lebensgeschichte« mitsamt einem Bericht über dessen Translatio und Ottos III. Besuch in Gnesen, deren Vorhandensein freilich auch mit der Adalbertsverehrung in Magdeburg zusammenhängen kann oder einen polnischen Hintergrund haben könnte. Deutlicher ist in dem Legendar der Bezug zu Böhmen in einer Wenzelslegende, die um ein Wunder erweitert wurde, bei dem 1391 der Heilige, im Gegenzug für das Versprechen, dass er dort verehrt werde, in Halle ein Feuer gelöscht haben soll, und in der Ludmillalegende Fuit in provincia, in der mit einem anderen Schluss versehenen Translatio s. Ludmillae (BHL 5028) und in einer verkürzten Bearbeitung der Vita der fünf Brüder (BHL 1148), die mit dem Schluss versehen wurde quattuor autem aliorum in Praga cum maximo honore seruantur et recoluntur.

Außerdem gibt es in dem Legendar eine Vita der hl. Hedwig

Die Vita des hl. Stephan befindet sich auf den Blättern $144^{\mathrm{vb}}-147^{\mathrm{vb}}$. Geschrieben von einer flüchtigen Hand, die mehrfach aufgrund von Augensprung Zeilen tilgen und an der richtigen Stelle nachtragen musste. Die übereinstimmende Auswahl ihrer Einträge mit der in der vorgenannten Prager Handschrift könnte auf eine gewisse gemeinsame Tradition hinweisen.

Handschriften vgl. B. Fleith, Studien zur Überlieferungsgeschichte der lateinischen Legenda aurea, Subsidia hagiographica 72, Bruxelles 1991. A. Vidmanová, Legenda aurea a Čechy, in: Jacobus de Voragine, Legenda aurea, Praha 1984, S. 11-64. ${ }^{11}$ Vgl. J. Klapper, »Hedwigis electa«. Eine Hedwigsvita aus dem Anfang des 14. Jahrhunderts, „Archiv für schlesische Kirchengeschichte" 19, 1961, S. 53-61. Die Vita, wie sie in der Hs P vorliegt, bricht mitten im Text ab (in der Ausgabe von Klapper S. 55 [Z. 19], um von der Vita Henrici regis Ungarie gefolgt zu werden. Sie ist mit Sicherheit nach 1300 entstanden. Klapper nimmt an, dass ihr Ursprung in den ersten Jahren des 14. Jahrhunderts zu suchen sei.

${ }_{12} \mathrm{Zu}$ dieser Handschrift hat sich maßgeblich Ursula Winter geäußert, deren Beobachtungen hier mitgeteilt werden sollen. U. Winter, K. Heydeck, Die Manuscripta Magdeburgica der Staatsbibliothek zu Berlin Preussischer Kulturbesitz, Teil 2: Ms. Magdeb. 76-168, Staatsbibliothek zu Berlin. Preussischer Kulturbesitz. Kataloge der handschriftenabteilung, hrsg. von E. Overgaauw, Erste Reihe: Handschriften Bd. 4, Die Manuscripta Magdeburgica Teil 2, Wiesbaden 2004, bes. S. 127-137; U. Winter, Das Legendarium Magdeburgense in der Staatsbibliothek zu Berlin - Preußischer Kulturbesitz (Mss. Magdeb. 26 u. 138.), in: Scrinium Berdinense: Tilo Brandis zum 65. Geburstag, hrsg. von Peter Jörg Becker, Berlin 2000, S. $320-327$. 


\section{Das Verhältnis der Handschriften zueinander.}

Die Handschriften O, P und M weisen im Wesentlichen denselben Textbestand auf. Sie sind aber durch zahlreiche Trennfehler untereinander gekennzeichnet, die es verunmöglichen anzunehmen, dass die ältere Handschrift $\mathrm{P}$ die Vorlage von O oder M wäre.

Insbesondere verbinden $\mathrm{M}$ und $\mathrm{P}$ zahlreiche Abweichungen gegenüber $\mathrm{O}$, die jedoch meistens nicht sehr signifikant sind, Beispiele dafür sind:

\begin{tabular}{|l|l|}
\hline O15,12 totum vero & vero totum P M \\
\hline O16,16 descendit & discessit P (auf Rasur) M \\
\hline O19,23 craa & traa P M \\
\hline O23,21 iube te de sollicitudine & iubeo te de sollitudine P M \\
\hline O28,14 necessarijs & nuncijs \\
\hline O28,26 mesko & mezko P M \\
\hline O28,26 duci polonorum & polonorum duci P M \\
\hline O29,7f mittere iam & iam mittere P M \\
\hline O34,11 meszkoni & mezkoni P M \\
\hline O35,11 eum turbatum & turbatum eum P M \\
\hline O43,34f per nepotem & per avunculum P M \\
\hline O46,15f om. & $\begin{array}{l}\text { ingemiscere et clamare audiret P }(\mathrm{Z}) ; \text { ingemiscere } \\
\text { clamare audiuit M }\end{array}$ \\
\hline O48,12 terram totam & totam terram P M \\
\hline O53,8 u. 35 Hemericus ${ }^{13}$ & Heinricus P; Hindericus M \\
\hline O59,8 deo & domino P M (= BHL 7921) \\
\hline O70,3 preparanda & prestanda (=BHL 7921) \\
\hline O72,38f per gentes & parentes (=BHL 7921) \\
\hline O73,1 viri suffragio confringentes & viri suffragio conferentes (P = BHL 7921) \\
\hline
\end{tabular}

${ }_{13}$ Diese Namensform ist für ungarische Quellen typisch, während Heinrichus, bzw. Hindericus auf einen (nord-)deutschen Verfasser / Schreiber hinweist. 
Dagegen trennen M zahlreiche und schwerwiegende Abweichungen von $\mathrm{O}$ und $\mathrm{P}$

\begin{tabular}{|l|l|}
\hline O15,6 plaga dei & plaga et flagellum dei \\
\hline $\begin{array}{l}\text { O16,38-17,15 P Dehinc pertransiuit alpes tyranice et } \\
\text { uenit in terminos dacie et slauonie (sclauonie P) et } \\
\text { conflictum magnum octo diebus }\end{array}$ & $\begin{array}{l}\text { Demum pertransiuit alpes et in terminos cruwatie } \\
\text { et sclavonie ubi conflictum magnum } 8^{\circ} \text { diebus } \\
\text { habuerunt }\end{array}$ \\
\hline O20,18 congregatis cunctisque principibus & igitur congregatis cunctis principibus \\
\hline $\begin{array}{l}\text { O20,24-30 Post mortem autem patris sui uxor } \\
\text { coloman genuit filium qui uocatus est bela }\end{array}$ & $\begin{array}{l}\text { Post mortem patris uxor coloman genuit filium } \\
\text { nomine Bela }\end{array}$ \\
\hline $\begin{array}{l}\text { O21,1 Inde autem mouens se uenit in terram } \\
\text { sclauoniam quam attauus suus ungariam appellauit }\end{array}$ & $\begin{array}{l}\text { post venit in terram Sclauoniam, quam attauus suus } \\
\text { ungariam vocavit }\end{array}$ \\
\hline O21,11 de regione polonie & de regno polonie \\
\hline $\begin{array}{l}\text { O21,22 hec cepit uirum suum ad christum conuertere } \\
\text { et fidem katholicam tenere et a cultura ydolorum } \\
\text { recedere }\end{array}$ & $\begin{array}{l}\text { Hec cepit yesse ad christum convertere et fidem } \\
\text { katholicam instruere et a cultura ydolorum secernere }\end{array}$ \\
\hline O22,19 Idem tempus celitus depositum & om. M. \\
\hline O23,22 P (ubi P) tibi... dominus & om. M \\
\hline $\begin{array}{l}\text { O24,7-15 Verumptamen uirum spirituali legacione } \\
\text { tibi transmittendum honorificabiliter suscipito } \\
\text { uenerabiliter habeto, exhortacionibus eius cordis } \\
\text { fidelem assensum }\end{array}$ & $\begin{array}{l}\text { Verumptamen virum spirituale legacione tibi } \\
\text { transmittendum honorifice suscipere et apertis } \\
\text { cordibus eiusque exhortationibus assensum prebeto }\end{array}$ \\
\hline O25,19 qui eam alloqui taliter cepit & alloquens eam dicens \\
\hline O26,1 P tamen ${ }^{14}$ & om. M \\
\hline O26,3 P uel & et M \\
\hline O31,3 P sine certatione & sine dilatione M (sine cunctatione in BHL 7921) \\
\hline O33,12f. romanae sedis pontifex & papa M \\
\hline $\begin{array}{l}\text { O36,29-31 siue poloni in vngaros, siue ungari } \\
\text { in polonos }\end{array}$ & Siue poloni in vngaros siue contrarium M \\
\hline
\end{tabular}

${ }^{14}$ Karacsonyi las hier cum, Kownacki und Endlicher emendierten tum, Piłat las in der ansonsten gleichlautenden Stelle bei $\mathrm{Z}$ tatsächlich tum. Die Abkürzung dürfte aber richtiger wie in $\mathrm{P}$ als tamen aufzulösen sein. 
Besonders umfangreich sind die Abweichungen von $\mathrm{M}$ gegenüber $\mathrm{P}$ und $\mathrm{O}$ auf fol. 146 $\mathrm{r}$ :

\begin{tabular}{|c|c|}
\hline $\mathrm{O} 38_{23}-43_{36}$ und $\mathrm{P}$ & M146rb \\
\hline $\begin{array}{l}\text { Post hec autem sancte dei genitricis semperque } \\
\text { uirginis marie incipitur officum salue sancta parens } \\
\text { iuxta missam uero rex (P rex uero) oleo sacro } \\
\text { inungitur et consecratur. } \\
\text { Post missam uero osculum pacis omnibus prebet } \\
\text { alii uero manum sacram anulum sacrum salutant et } \\
\text { benediccionem ab eo recipiunt. } \\
\text { Quo finito presules cum clero comites cum populo } \\
\text { Kyrieleyson cum congruis laudibus proclamant, deum } \\
\text { omnipotetem et sanctos apostolos Petrum et Paulum } \\
\text { benedicunt, quod sanctus deo dilectus Stephanus } \\
\text { exunccione sacri crismatis perunctus }\end{array}$ & $\begin{array}{l}\text { Post hec infra missarum solempnia rex stephanus } \\
\text { oleo sacro inungitur }\end{array}$ \\
\hline $\begin{array}{l}\text { Dyademate regalis dignitatis feiliciter est } \\
\text { coronatus. Post acceptum regalis excellencie lignum } \\
\text { (signum?) (P dig }{ }^{\mathrm{m}} \text { dignum / dignitatem?) tam } \\
\text { epicsopales ecclesias amplians regaliter disposuit }\end{array}$ & $\begin{array}{l}\text { et dyademate regalis dignitatis feliciter est } \\
\text { coronatus. Post acceptam regalis excellencie } \\
\text { dignitatem tam episcopales et ecclesias quantum alias } \\
\text { regaliter amplians disposuit }\end{array}$ \\
\hline $\begin{array}{l}\text { quam crucibus et uasis aliis supellecitilibus ad } \\
\text { ministerium dei pertinentibus secundum quod } \\
\text { unicuique opus erat sufficienter decorauit. }\end{array}$ & $\begin{array}{l}\text { cum crucibus et uasis aliisque suppellectibus ad } \\
\text { ministerium dei pertinentibus secundum quod } \\
\text { unicuique opus erat sufficienter decorauit }\end{array}$ \\
\hline $\begin{array}{l}\text { Post officium sancte trinitatis incipitur Benedicta sit } \\
\text { sancta trinitas officia uero sancta pro rege et principe } \\
\text { mezkone et pro cuncto populo per presulem astritum } \\
\text { offeruntur pace accepta et missa finita ad tentoria sua } \\
\text { redeunt ubique in gaudio et leticia epulis et potibus } \\
\text { in cordis et organis et tympanis et choris cytharis et } \\
\text { phyalis }\end{array}$ & $\begin{array}{l}\text { post haec officium de sancta trinitate incipitur scilicet } \\
\text { benedicta etc. pro rege et principe mezkone et pro } \\
\text { cuncto populo pro formanda pace, qua missa finita ad } \\
\text { tentoria sua redeunt ubi in gaudio et leticia epulis et } \\
\text { potibus ac musicis variis instrumentis }\end{array}$ \\
\hline $\begin{array}{l}\text { auunculus et nepos letos octo duxerunt dies omnisque } \\
\text { polonorum exercitus a maiore usque ad minorem } \\
\text { muneribus replentur duci uero multa bona per } \\
\text { nepotem (P: avunculum) offeruntur. }\end{array}$ & $\begin{array}{l}\text { avunculus et nepos letos } 8 \text { duxerunt dies omnisque } \\
\text { polonorum exercitus a maiore usque ad minorem } \\
\text { muneribus replentur duci uero multa bona per } \\
\text { avunculum offeruntur. }\end{array}$ \\
\hline
\end{tabular}


Weitere trennende Abweichungen von $\mathrm{M}$ gegenüber $\mathrm{O}$ und $\mathrm{P}$ sind in

\begin{tabular}{|c|c|}
\hline $\mathrm{O}$ (und $\mathrm{P}$ ) & M \\
\hline $\begin{array}{l}44,17-23 \text { in ipsa regalis sedis ciuitate, que alba } \\
\text { nuncupatur, sub laude et titulo genitricis dei famosam } \\
\text { et grandem ecclesiam opere mirifico construere cepit }\end{array}$ & $\begin{array}{l}\text { in ipsa regalis sedis ciuitate witzeborch, que alba } \\
\text { dicitur, sub laude et titulo marie virginis famosam } \\
\text { ecclesiam opere mirifico construere fecit }\end{array}$ \\
\hline $\begin{array}{l}47,12-22 \text { Quadam igitur nocte per reuelacionem } \\
\text { qua }<\mathrm{n}>\text { dam infra diem et noctem ad albam } \\
\text { transsiluana }<\mathrm{m}>\text { precepit festinare et omnes in rure } \\
\text { manentes ad munitas ciuitates, quam citissime posset } \\
\text { congregare }\end{array}$ & $\begin{array}{l}\text { Quadam igitur nocte per revelacionem quandam } \\
\text { precepit ut omnes in rure manentes ad munitas } \\
\text { ciuitates quam citissime possent festinarent }\end{array}$ \\
\hline $48,12 \mathrm{f}$ terram totam devastauit & totam terram Transsiluanam. \\
\hline $48,14 \mathrm{om}$. & albe civitate devastata \\
\hline 52,3 quid uidisset & si quem vidisset \\
\hline $52,5-7 \mathrm{om}$. & cui tum omnia visa retulit \\
\hline 52,9f constat, quod illum impletum est & erat impletum, quod actis apostolorum \\
\hline 52,13 in regnum caelorum & etc. \\
\hline $\begin{array}{l}52,17-19 \text { tribus annis in (in om. } \mathrm{P}=\mathrm{BHL} \text { 7921) } \\
\text { infirmitate continua }\end{array}$ & per triennium infirmitate continua \\
\hline $\begin{array}{l}53,3 \text { abortam }(\mathrm{P} \text { obortam }=\text { so korrigierten auch alle } \\
\text { Herausgeber vor Karacsonyi) }\end{array}$ & exortam \\
\hline 53,3 genitor & pater \\
\hline 55,2 vix umquam & vix ut numquam \\
\hline 57,12 f non.... plantaret & om. \\
\hline 58,11 inunctionem & unctionem \\
\hline 58,12 quibus ultimum benedicans & quibus expletis omnes benedicans \\
\hline 58,18 iesu christe & om. \\
\hline 59,5 cantu & planctu \\
\hline 59,10 monumento & sepulcro \\
\hline $59,22 \mathrm{f}$ per angelorum choros et laudantes & per angelorum choros laudans \\
\hline 59,29 sanctus & om. \\
\hline $60,5 \mathrm{om}$. & et voces \\
\hline 70,3 f germanum & fratrem \\
\hline 71,10 miseretur et stabat & misereretur instabat (=corr. Ketrzynski) \\
\hline 71,26 annuente & fauente \\
\hline 72,13 obsessis & obsessi (=BHL 7921) \\
\hline $72,18-28$ Unde... collaudarent & om. \\
\hline 72,38 accepit & accepit quod et factum est \\
\hline 73,1 viri suffragio confringentes (conferentes $(\mathrm{P})$ ) & viri suffragio rogantes \\
\hline
\end{tabular}


Ferner verbinden $\mathrm{M}$ Lesungen mit $\mathrm{Z}$, die zugleich von $\mathrm{P}$ und $\mathrm{O}$ trennen:

\begin{tabular}{|l|l|}
\hline O und $\mathrm{P}$ & $\mathrm{Z}$ und M \\
\hline O17,1 dacie & chrvacie (cruwatie M) \\
\hline O17,10 om. & $\begin{array}{l}\text { conflictum magnum } 8^{\circ} \text { diebus habuerunt M fecerunt } \\
\text { conflictum magnum octo diebus }\end{array}$ \\
\hline O31,6 P cito & scito MZ \\
\hline O38,9 P plurimorum & privilegiorum MZ \\
\hline O46,10 P pro grege & pro regno MZ \\
\hline O58,11 P inunctionem & unctionem MZ \\
\hline
\end{tabular}

Das Verhältnis der Handschriften zueinander ist nicht abschließend zu klären. Keine kann die unmittelbare Vorlage der anderen gewesen sein, auch wenn man annimmt, dass $\mathrm{O}$ und $\mathrm{M}$ getreuliche Abschriften einer älteren Handschrift gewesen wären.

Näher miteinander verwandt sind jedenfalls die Hss. P und $\mathrm{O}$, die einen fast identischen Text vorweisen, der nur von geringen Abweichungen gestört wird, die gleichwohl nicht zulassen, dass $\mathrm{P}$ die Vorlage von $\mathrm{O}$ gewesen sein kann. Freilich kann eine gemeinsame Vorlage postuliert werden. Die Verwandtschaft der beiden Hss. wird noch dadurch unterstrichen, dass jeweils wenigstens auch die Vita des hl. Emmerich in einem nahezu gleichen Wortlaut vorliegt ${ }^{15}$.

Bemerkenswert ist dabei, dass P die Vita des hl. Stanislaw nicht enthält. Entweder war sie schon in der Vorlage von P nicht enthalten und wurde erst später hinzugefügt, oder aber sie wurde später aus Mangel an Interesse an diesen polnischen Angelegenheiten ausgelassen.

Das Verhältnis von $M$ und $P$ ist weniger deutlich zu bestimmen. Grundsätzlich weicht $M$ durch seine zahlreichen Auslassungen, Ergänzungen und Änderungen stark von P und natürlich auch von $\mathrm{O}$ ab, nicht wenige davon könnten sich gewiss auf die Flüchtigkeit des sicher norddeutschen Schreibers zurückführen lassen, andere dann aber wieder auf seine (bzw. des Schreibers seiner Vorlage) Fähigkeit zur sinnvollen (ergänzenden) Erläuterung. $\mathrm{M}$ und $\mathrm{P}$ hat darüber hinaus gegenüber $\mathrm{O}$ einige wenige verbindende Abweichungen, von denen einige auch eine größere Nähe zu BHL 7921 dokumentieren. Genauso gibt es einige signifikante verbindende Abweichungen von $\mathrm{M}$ und $\mathrm{Z}$, namentlich die gemeinsame Verwendung des Namens cruwatia gegenüber dem dacia der beiden anderen Hss.

Während bisher alle Handschriften aus Polen stammten, lenken die beiden neuen Manuskripte den Blick auf ein mit Böhmen verbundenes Umfeld und machen deutlich, dass diese Version der Vita bzw. der Chronik auch jenseits der polnischen Grenzen auf Interesse stieß und dass sie vermutlich weitaus weiter verbreitet gewesen sein könnte, als bisher angenommen. Auch lässt sich ihr Entstehungsdatum weiter eingrenzen, setzt doch die Hs $\mathrm{P}$ einen eindeutigen terminus ante, der letztlich mit der bisher ältesten bekannte Hs Z zeitgleich, oder sogar noch älter ist.

\footnotetext{
15 BHL 2529b: O (=D in AASS Nov. II Pars I 487B, = L in Scriptores rerum hungaricarum tempore ducum regumque stirpis arpadianae gestarum, ed. I. Szentpétery, Bd. 2, S. 449-460, hier S. 449): „Gloriosus rex primus Stephanus ducatum Ungarie tenuit annis octo, regnavit autem triginta tribus annis, mensibus septem, diebus XIIII; migravit autem ad Dominum anno incarnacionis Domini $\mathrm{m}^{\circ} \mathrm{ccc}^{\circ} \mathrm{IIII}^{\circ}$ (sic), decimo octavo kal. septembris, feria quinta; cuius venerabile corpus Albe reconditum est; per huius mellifluam prudenciam novissimis, ut dicam, temporibus tocius Panonie regnum lumine veritatis agnovit. $\mathrm{P}=$ Gloriosus rex primus Stephanus ducatum Ungarie tenuit annis octo regnauit autem xxxiiius annis mensibus vii diebus xiiii, migrauit autem ad dominum Anno domini incarnationis mo xxxiiiio xviii kal. septembris feria va cuius venerabile corpus albe recordatum est, per huius mellifluam prudenciam nouissimis ut dicam temporibus tocius pannonie regnum lumen veritatis agnouit".

Vgl. zu dem Problem der polnischen Emmerich-Legende auch I. Toth Sarolta, Magyar és lengyel Imre-legendák, Acta Universitatis Szegediensis. Acta Historica XI, Szeged 1962, S. 5 und 57-70.
} 
Der Hintergrund der Aufnahme der ungarischen Heiligen in eine böhmische Legenda aureaHandschrift lässt sich letztlich nicht klären. Doch ist auch angesichts der Rezeption der ungarischpolnischen Chronik in den Klöstern Kamenz und Heinrichau ${ }^{16}$ und aufgrund der Integration des Hedwigsstoffes an eine schlesische Verbindung, konkret an eine Verbindung mit den Zisterziensern zu denken, die die Verbreitung der Verehrung der hl. Hedwig ${ }^{17}$ beförderten.

Dies wiederum würde nahelegen, dass die ungarisch-polnische Chronik bereits unter der Regierung Wenzels II. (1278-1305) epitomiert worden wäre, der insbesondere Kamenz reich mit Besitz ausgestattet und auch auf andere Weise bevorzugt behandelt hatte ${ }^{18}$.

\section{Nachtrag}

Eine weitere Handschrift ist mir erst nach Abschluss der Arbeiten an den vorliegenden Bemerkungen durch Herrn Dr. László Veszprémy zugänglich gemacht worden. Es handelt sich dabei um eine auf das Jahr 1435 datierte Hs. aus der Abtei Sieciechów (S), die teilweise durch Wasserschaden nicht oder nur schwer lesbar ist (Nationalbibliothek, Warschau 3316 - fol. 196 $\left.{ }^{\mathrm{v}}-199^{\mathrm{r}}\right)^{19}$.

Die Handschrift $\mathrm{S}$ ist weitestgehend mit der bekannten Hs. O identisch. Gleichwohl weisen die Abweichungen darauf hin, dass sie auf einer besseren Vorlage basierte, ohne dass anzunehmen wäre, dass sie selbst die Vorlage von O sein könnte.

So bestätigt die Lesung traa gegen craa O19,23 die Lesungen in P und M. Ähnliches findet man auch, wenn etwa alle Handschriften einschließlich S transmisit gegenüber transiuit in O47,3 lesen. $\mathrm{S}$ bekräftigt auch die Lesung parentes von $\mathrm{P}$ und $\mathrm{M}$ gegenüber dem offensichtlich fehlerhaften per gentes in O71,38f. Während diese Abweichungen dafür sprechen, dass der Schreiber von $\mathrm{S}$ seine Vorlage besser abschrieb als der Schreiber von O lassen die folgenden Unterschiede darauf schließen, dass S kaum die unmittelbare Vorlage von O gewesen sein kann: S hat ferner richtig signum, während O40,6 lignum schreibt, was schon Kętrzynski dementsprechend korrigierte (aufgrund der erheblichen Abweichungen in O, P und M scheint diese Stelle schon in der gemeinsamen Vorlage schwer lesbar gewesen zu sein). Anstelle des unsinnigen descendit in O16,16 schrieb S ferner disperavit (PM: discessit). Nicht zu erklären ist, dass S anstelle von preparanda O70,3 (PM prestanda) predicanda schrieb. Schließlich ist zu bemerken, dass $\mathrm{S}$ anders als $\mathrm{O}$ an der germanischen Namensform Henricus festhält, für die $\mathrm{O}$ die ungarische Form Emericus verwendet hat.

\footnotetext{
${ }^{16}$ R. Grzesik, Kronika wegiersko-polska, S. 103-108; A. Quéret-Podesta, The influence of the so-called Hungarian-Polish chronicle on the Silesian medieval chronicles, „Slezský sborník”, 108, 2010, S. 273-278.

${ }_{17}$ K. Charvátová, Déjiny cisterckého řádu v Čechách 1142-1420. Kláštery na hranicích a za hranicemi Čech, Praha 2009, S. 134-136; J. Gottschalk, St. Hedwig und der Zisterzienserorden, „Archiv für schlesische Kirchengeschichte”, 25, 1967, S. 38-51. Zur Hedwigsverehrung in Böhmen vgl. Ders., St. Hedwig, Herzogin von Schlesien, Forschungen und Quellen zur Kirchen- und Kulturgeschichte Ostdeutschlands 2, Köln 1964, S. 302-304, ein frühes Dokument ist dabei ein Lektionar des Jahres 1316, das aus dem Benediktinerkloster Opatovice [Opatowitz] stammte, das über die Entsendung von Mönchen in eine Propstei in Wahlstatt und dann in Grüssau sehr eng mit Hedwig und den schlesischen Geschicken verbunden war. Im Oktober 1357 stiftete Bohuslav, der Propst von Leitmeritz, Bruder des Prager Erzbischofs Arnestus, Kanoniker von St. Ägidius in Breslau und Kaplan Kaiser Karls IV. einen Altar zu Ehren der hll. Hedwig und Anna wohl in Rücksicht auf Anna von Schweidnitz, die Frau Karls IV. vgl. auch H. Manikowska, Legenda św. Jadwigi-obieg i transformacja, in: Kultura elitarna a kultura masowa w Polsce późnego średniowiecza, hrsg. von B. Geremek, Wrocław 1978, S. 155-171.

18 30. April 1294: Schenkung der Stadt Mittelwalde / Międzylesie; 26. März 1298: Schenkung von Stalsdorf bei Freudenthal / Bruntál als Ersatz für Trebenowice, das er während seiner Minderjährigkeit, also vor 1288, unrechtmäßig geschenkt hatte. Regesta dipolmatica nec non epistolaria Bohemiae et Moraviae, p. 2: annorum 1253-1310, Red. J. Emler, Pragae 1882 , s. 705 № 1643; s. 769 № 1788. - K. Charvátová, Dějiny cisterckého řádu v Čechách 1142-1420. Kláštery na hranicích a za hranicemi Čech, Praha 2009, S. 134-136. - H. Grüger, Die zisterziensische Architektur in Schlesien in den Jahren 1200-1330. Bemerkungen zu Marian Kutzner, Cysterska architektura na Śląsku w latach 1200-1330, „Archiv für schlesische Kirchengeschichte", 29, 1971, S. 1-31, hier 26.

${ }_{19}$ Fleith, Studien, Nr. 961.
} 


\section{Trzy nowe rękopisy tzw. Kroniki węgiersko-polskiej}

Dotąd znany był tylko jeden rękopis (O) krótszej redakcji tzw. Kroniki węgiersko-polskiej (BHL 7922), pochodzący z XV w. Niniejsze studium dołącza znaleziska trzech nowych przekazów (M, P, S), z których najstarszy (P) datowany jest na połowę XIV w., a dwa pozostałe na XV w. Szczególnie rękopis $(\mathrm{M})$ różni się w wielu miejscach od wersji $(\mathrm{O})$. W świetle tych ustaleń można przyjąć, że Kronika węgiersko-polska została skrócona już za rządów Wacława II (1278-1305), który utrzymywał bliskie kontakty ze śląskimi cystersami. Ponownego rozważenia wymaga także historia redakcji dłuższej wersji Kroniki węgiersko-polskiej.

Kontakt do autora: albrecht@rgzm.de 